\title{
I'll Have Fries with That: Increasing Choice Complexity Promotes Indulgent Food Choices
}

\author{
Yong Kyu Lee \\ York College, The City University of New York \\ Kimberlee Weaver \\ Virginia Tech
}

Stephen M. Garcia

University of Michigan

\begin{abstract}
Three studies showed that the way that options are presented in a choice set-as combinations of intersecting attributes or in a more sequential "a la carte" choice format-affects the degree to which consumers adhere to their goals in the consumption setting. Specifically, using the context of food consumption and healthy eating, results showed that consumers were more likely to make double indulgent choices, the choice of both an indulgent entrée and an indulgent side item, when choosing from a menu consisting of predetermined "combination meals" than when selecting among the same entrée and side options in an a la carte fashion. Studies 2 and 3 implicated a goal distraction mechanism in driving the effect; the combination format, with its cross-cutting of product choices into various combinations, reduces the salience of goal-related constructs on implicit measures. In showing that different product presentation formats can affect the degree to which consumers make goal-consistent choices, the current work adds to work on the effects of environmental influences on goal progress and goal achievement. Implications for encouraging goal-consistent behavior in the context of healthy eating as well as other important consumer goal contexts are discussed. $\odot 2016$ Wiley Periodicals, Inc.
\end{abstract}

Whether at a sit-down or fast-food restaurant, the menu items often include "meal" or combination choices. For instance, at McDonald's, there are a number of "value meals" from which one can choose. While these meals and combination plates make it easier for consumers to choose by predetermining what pairs together well, does this choice among combinations make it more likely that consumers will indulge in unhealthy food items compared to a situation where they chose between the same options one-at-a-time, using an "a la carte" decision-making process? For example, would someone be more likely to order a combo meal that included a cheeseburger and fries if the person is making a choice among "meals" than if they had to choose a kind of sandwich first and then a pairing side next?

The current analysis argues that consumers become distracted from their goals-in the example above goals for weight management-when choosing among a large number of options in which there are varying and intersecting attributes relative to situations where the decision is simplified through sequential choices on key attributes. Thus, the current paper posits that in the consumer context of food, the way that food options are presented on the menu-as "meals" versus as "a la carte" choices-will differentially impact their consumption of healthy versus indulgent items.

Across three studies, the current findings offer support for this goal distraction account by showing that consumers are more likely to make double indulgent choices-a choice of both an indulgent entrée and an indulgent side-when the selections are presented as a choice between meals or combinations than when the identical selections are presented in an a la carte choice format. The current studies also begin to explore the mechanism underlying this effect, showing that it is stronger for consumers who have goals in the decision context (e.g., weight management goals) versus not and that the combination presentation condition reduces the cognitive accessibility of concepts related to the goal on implicit measures. In doing so, the present analysis extends past work on goal progress and goal achievement by investigating how a ubiquitous but 
relatively understudied contextual factor in the consumer behavior literature - how a choice from a large number of options with overlapping attributes versus the same decision in a simplified sequential choice format-affects consumers' ability to pursue their goals.

\section{THEORETICAL BACKGROUND}

\section{Goal Distraction: When Too Many Options Undermine Goal Pursuit}

Making a simple choice can sometimes be easy and sometimes difficult. Easier choices might include whether a person prefers a pen with black or blue ink, or red or white wine. However, even these rather simple choices can become somewhat more difficult to make as the complexity of the options and the number of options is increased. For example, now consider the following: Would you prefer a black ballpoint pen, black ink tip pen, a blue ballpoint pen, or blue ink tip pen? Red Merlot wine, red Cabernet wine, white Riesling wine, or white Sauvignon Blanc wine? The two latter decisions are arguably less easy to make than the two former. In the backdrop of this more difficult decision context, the current paper aims to build the hypothesis that pursuing one's presumed goals will also become more difficult.

Several literatures can help to build this goal distraction account, and the literature on choice overload is a good place to start. In one classic study, either six or 24 varieties of jam were set up on a display stand at an upscale supermarket in Menlo Park, California. Results showed that, while shoppers were more willing to stop and sample the jams when there were 24 rather than six varieties available, they were more likely to actually purchase a jam when there were only six on display (Iyengar \& Lepper, 2000). As the authors described, consumers were less likely to purchase a jam in the large variety condition because the large size of the choice set was overwhelming. In addition, one might infer that the large number of options distracted consumers from a presumed goal of buying their favorite jam. A follow-up study in this vein makes the link between goal pursuit and choice set size more directly. Obviously, workers in corporate America, especially those working for companies with $401 \mathrm{k}$ programs, know that saving for retirement is important. In other words, saving for retirement is an important goal. However, past work has shown that employee participation in $401 \mathrm{~K}$ retirement programs actually decreases as the number of investment options offered in a given plan increases (Iyengar, Huberman, \& Jiang, 2004). In both these studies, the sheer number of options in a choice set undermined people's ability to accomplish the ostensible goal at stake (e.g., choosing an investment strategy; Broniarczyk \& Griffin, 2014).
Just as the literature on choice overload suggests that the number of options can undermine goal pursuit, the literature on brand confusion points to a similar phenomenon-as the number of brands to choose from increases, people become less able to choose their favorite brand (Foxman, Berger, \& Cote, 1992; Huffman \& Kahn, 1998; Mitchell, Walsh, \& Yamin, 2005). For example, in one study (Jacoby, Speller, \& Kohn, 1974), participants specified the attributes that would constitute their "ideal" laundry detergent, thus establishing an idiographic standard of an "ideal" detergent for each participant. Participants then selected an option for purchase from a choice set of 4,8 , or 12 possibilities where the number of pieces of information about each brand was also manipulated. Results showed that participants became increasingly less able to choose their "ideal" detergent as the number of options increased. Similar effects were found in a followup study that varied the presentation order of the attribute information and the degree to which attributes overlapped among options (Jacoby, Speller, \& Berning, 1974). Thus, the literature on brand confusion also suggests that a large number of options as well as differing amounts of overlapping options can undermine goal pursuit.

Interestingly, recent work shows that such confusion and distraction can even happen at the perceptual level when too much information is provided. Jia, Shiv, and Rao (2014), for instance, showed that consumers perceived more similarity between two pairs of sunglasses and were more likely to defer their purchase when they saw multiple photos of each pair from different angles than when they saw only one photo of each.

\section{Minimizing Goal Distraction: Simplifying the Choice by Sequential Decisions}

While past work suggests that choosing from a large choice set of options can foster goal distraction, then simplifying the choice through sequential decision making should minimize goal distraction. This possibility is especially interesting because it predicts a different effect for goals even though all possible combinations that are present in the large choice set of options are also present in the simplified sequential decision process. Indeed, previous work on mass customization (Huffman \& Kahn, 1998) supports this possibility. In mass customization contexts, people can design their own product (i.e., sofa). However, the sheer number of possibilities and combinations among them can create confusion and undermine customer satisfaction when customers have to choose every single attribute without any structure. In contrast, when the decision is simplified by sequentially asking customers about their key attributes and preferences within them, then there is less confusion and higher customer satisfaction. Although not looking at adherence to or distraction from goals per se, this work is suggestive in that it highlights that the simplified sequential 
choice format allows consumers to focus their attention spotlight more on what is important in the choice set.

Another consequence of simplifying the choice through sequential decision making is that the tradeoffs between options inherently become more stark. For example, although consumers in a between-subjects design rated indulgent desserts more highly than healthy alternatives, they actually tended to choose the healthy alternative when it was presented in a choice set sideby-side with an indulgent option. Thus, the stark choice set simplifies the decision (Okada, 2005) and, in this case, promotes the pursuit of the implicit healthy eating goal. Work by Sela, Berger, and Liu (2009) also gives credence to the possibility that a simplified decision setting can promote goal pursuit. They found that $55 \%$ of people chose a fruit (healthy item) over a cookie (indulgent item) when one option of each was presented. However, $76 \%$ chose a fruit when the salience of the healthy/indulgence distinction was highlighted even further in the choice setting by presenting several options on two separate and opposing "sides" of a platter, with six types of fruit on one side and six types of cookies on the other. In this latter case, the distinction between the healthy and indulgent attribute is made even more salient, and people became more likely to choose the healthy items in accord with their implicit health goals.

These results are consistent with past work on goals that shows that even when consumers have set goals for themselves (e.g., healthy eating goals), whether they will actually exhibit goal-consistent behavior is often affected by environmental cues (Herman \& Polivy, 1983; Kruglanski et al., 2002; Papies \& Hamstra, 2010; Stroebe, Mensink, Aarts, Schut, \& Kruglanski, 2008). For instance, while dieters set goals to eat fewer calories, they often fail to follow through. One way to increase goal-consistent behavior is through environmental cues that cognitively activate the goal, like a low-calorie cookbook (Kruglanski et al., 2002). Thus, the "divided platter" condition in Sela et al. (2009) may have served as a cue to remind consumers of the implicit healthy eating goal. Just as increased salience increases adherence to goals, the distracting effects of intersecting options can lower the salience of the same goals relative to an a la carte setting and in turn decrease adherence to them.

Thus, to extrapolate from past work onto the current question of interest, relative to a large choice set of options with intersecting attributes, which should distract from a goal and reduce the salience of it, simplified sequential choices along key attributes should facilitate goal pursuit by heightening the salience of goals, and thus remind or cue consumers to make goal-consistent choices. If so, then consumers should make relatively fewer goal-consistent choices in the combination condition than the a la carte choice one, even though the exact same option possibilities are present in both cases, only the format of the choices is changed.

\section{HYPOTHESES}

Synthesizing this goal distraction account based on the literature reviewed above, the current paper thus posits the following hypotheses:

H1: Consumers will be less likely to make choices consistent with their goals when choosing from a large number of options that intersect underlying attributes, compared to situations where they are making simplified sequential decisions.

While Hypothesis 1 captures the predicted behavioral effect, Hypothesis 2 focuses on the goal distraction mechanism. It predicts that consumers become distracted from their goals when choosing among a large number of options with intersecting features, compared to situations where they are making simplified sequential decisions that highlight one feature at a time. Of course, critical to this distraction mechanism is the role of goals. That is, according to the goal distraction account, the behavioral effect of Hypothesis 1 should only be observed among consumers who actually have goals that are relevant to the decision context (i.e., weight management goals, etc.); that is, goals must be present in order to have goal distraction. On the other hand, if consumers do not have any goals relevant to the decision context, then no differences between the choice presentation formats should be observed because there are no relevant consumer goals to distract.

This prediction is consistent with previous work on goals, which shows that while people with goals toward a given behavior are strongly affected by environmental stimuli that cue those goals, people without goals for that same behavior are unaffected by the same environmental stimuli (Kruglanski et al., 2002; Papies \& Hamstra, 2010; Stroebe et al., 2008). In one demonstration, for instance, consumers with goals to manage their weight chose an apple over a chocolate bar more when primed with a low-calorie cookbook than in a no prime control condition, but the choices of those who did not have weight management goals were unaffected by the low-calorie prime and were presumably driven by their preferences instead (Kruglanski et al., 2002). In a similar vein, Papies and Hamstra (2010) showed that while people with weight management goals ate less after seeing a poster with low-calorie recipes than after seeing a poster without a weight-relevant message, those without weight management goals ate the same amount regardless of the prime. Because the combination meal versus a la carte setting of interest here is also an environmental variable that affects the salience of healthy eating goals, based on this previous work on goals, Hypothesis 2 predicts that it should affect food choices only for those with active weight management goals and should have correspondingly no effect on the food choice preferences for those without such goals.

To be sure, this goal distraction account does not deny the fact that goals often help consumers make decisions in line with their goals; indeed, goals often help 
people stay focused. Instead, it simply suggests that consumers can become distracted from these goals in the backdrop of choosing from among many options of varying combinations of key attributes compared to situations where they make simplified sequential choices one key attribute at a time. Thus, Hypothesis 2 posits the following:

H2: The combination versus a la carte distinction is an environmental cue that affects the salience of healthy eating goals. Thus, consistent with past work, the food choices of people with goals should be more affected by it than those without such goals.

As outlined above, the process through which the large number of intersecting options in the combination meal condition is postulated to exert its distracting effect on food choice is by reducing the salience of cues or reminders of the healthy eating goal. That is, relative to the stark presentation of the a la carte food choice format, the large number of intersecting and cross-cutting options mixed together in the combination format should distract from the healthy eating goal by making it recede into the background. If so, then concepts that are related to the goal should be less cognitively accessible following the presentation of a combination meal menu compared to an a la carte format. Past work on goals has demonstrated the utility of response time measures in measuring the accessibility of goal-related concepts in other contexts (e.g., Fishbach, Friedman, \& Kruglanski, 2003; Kruglanski et al., 2002; Stroebe et al., 2008). Thus, Hypothesis 3 posits the following:

H3: Concepts relating to healthy eating goals should be less accessible following presentation of the combination meal format relative to presentation of the same product choices in a more simplified and sequential a la carte presentation one.

\section{OVERVIEW: THE PRESENT RESEARCH}

Because healthy eating is such an important issue in society, the current paper tests these hypotheses in the context of food selection by examining the extent to which consumers ultimately select indulgent versus healthy food items. In doing so, it focuses explicitly on decisions of a main dish (healthy vs. indulgent) and side dish (healthy vs. indulgent), and compares what consumers choose when presented with a choice of meals whose options represent all possible combinations (i.e., healthy main/healthy side dish, indulgent main/indulgent side dish, healthy main/indulgent side, indulgent main/healthy side) to what they choose when presented with those same options in a simplified sequential "a la carte" decision format (i.e., healthy or indulgent main dish, healthy or indulgent side).
Of course, the compelling feature of this design is that all possible combinations of options in the choice of meals are present in the sequential "a la carte" choices. The main dependent variable of interest was consumers' choices of double indulgent options-cases where a consumer chooses both an indulgent main dish and an indulgent auxiliary item-because double indulgent choices are the most consequentially harmful to people's overall health and well-being. The main predictions were that consumers would choose more indulgent options in the combination meal condition than in the starker and more simplified a la carte format (Hypothesis 1), that the difference between the two formats would be more pronounced for people who actually held weight management goals (Hypothesis 2), and that the combination format would reduce the cognitive accessibility of goal-related concepts on implicit measures relative to the a la carte presentation format (Hypothesis 3).

\section{STUDY 1: THE HAMBURGER STUDY}

Participants chose one burger and one side item either from a menu consisting of predetermined "combo meals" or from a menu where the same main dishes and sides were presented as two separate a la carte decisions. The key dependent variable was the frequency with which participants made a double indulgent choice, that is, a choice of both an indulgent main dish and an indulgent side. In line with Hypothesis 1 , the main prediction was that participants would be more likely to choose such an indulgent/indulgent combination when the items were presented as meals compared to when the same items had been presented in the starker a la carte fashion.

\section{Participants}

One hundred twenty-one adult participants $\left(M_{\text {age }}=30\right.$ years, $46 \%$ female) were recruited from the online panel Mturk.

Participants imagined they were shopping for lunch at a local restaurant and were looking at the menu where they could select a burger and side item combination. Four burgers were used as stimuli. Two were healthy (a veggie burger and a black bean burger) and two were indulgent (a bacon cheese burger and a BBQ burger). In addition, two side items were available: a salad (the healthy item) and French fries(the indulgent item). All stimuli were presented pictorially.

\section{Meal versus A La Carte Presentation}

In the meal condition,the burgers and side items were combined and shown together as a series of eight predetermined combinations on one menu. Thus, participants selected one meal among the eight possible meal combination options. In the a la carte choice condition, 


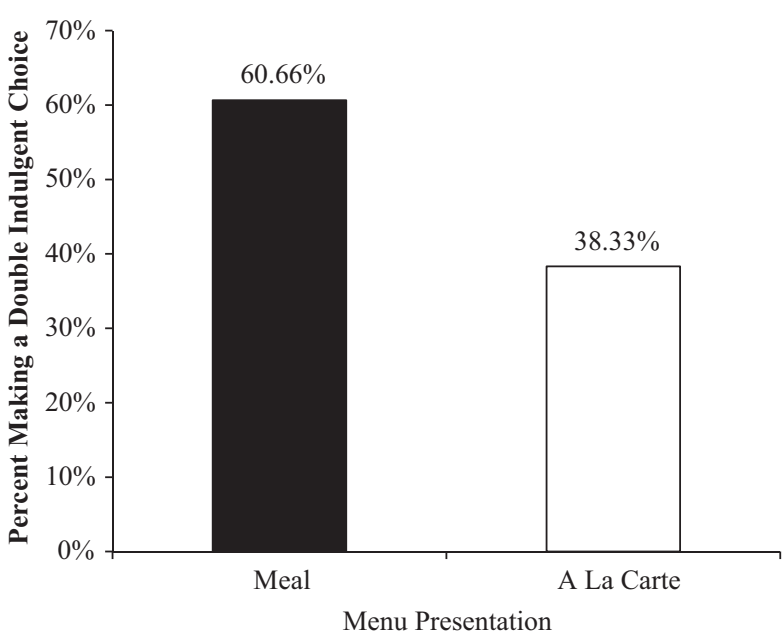

Figure 1. The effect of menu presentation (meal vs. a la carte) on the choice of a double indulgent meal-study 1 .

Note: A double indulgent choice is defined as a choice of both an indulgent main dish and an indulgent side.

participants first saw a burger menu and chose one of the four possible burgers and then saw a side menu where they chose one side item from the two options presented.

\section{Dependent Measures}

In addition to choice, the following control variables were also measured: participants' satisfaction with their selection; the degree to which they thought their selection was healthy or not; how often participants ate that type of item; and how much they liked the option they chose $(1=$ not at all, $7=$ very $)$.

\section{Results and Discussion}

The key dependent variable was the percentage of consumers choosing a double indulgent option (i.e., both an indulgent main dish and an indulgent side item) in the two menu presentation conditions. A choice was coded as a "1" if participants selected an indulgent option for both choices (e.g., bacon cheese burger and French fries) and a " 0 " otherwise. A logistic regression model showed, as predicted, participants presented with the options in the meal menu format made more indulgent choices than those presented with the identical food items in the a la carte menu format. Specifically, $60.66 \%$ of the participants selected an indulgent burger and an indulgent side item when they chose from a menu that presented the options as meals, while only $38.33 \%$ of the participants selected an indulgent main dish and an indulgent side item when the identical food options were presented in an a la carte format $\left(\chi^{2}=5.93, p<\right.$ 0.02) as shown in Figure 1.

The effect of menu presentation on consumers' tendency to make indulgent choices remained significant when controlling for the control variables (participants' satisfaction with their selection, their perceptions of the healthiness of their choice, the frequency with which they reported eating that type of food item in their daily lives, and how much they liked that item) as covariates $\left(\chi^{2}=8.11, p<0.005\right)$. As expected, none of those variables were themselves affected by the menu presentation variable (all $p \mathrm{~s}>0.70$ ). Table 1 presents the percentage of consumers choosing each of the possible main dish and side item combinations.

While Study 1 provides initial support for Hypothesis 1 that presenting options in a combination "meal" format can present a distraction from health goals and lead people to choose more indulgent food items than presenting the same options in an a la carte menu format, it did not actually measure weight or health goals. Instead, Study 1 only presumes that such goals are commonly held among a majority of people in the sample. Study 2 thus measures goals more explicitly.

\section{STUDY 2: IMPLICATING THE GOAL DISTRACTION ACCOUNT}

While Study 1 shows that consumers are less likely to make choices in line with their goals when they choose from a menu that presents food options as a series of combinations than when they choose among the identical items in a starker sequential decision-making format, Study 2 sought to conceptually replicate the effect in a different food context and simultaneously begin to implicate the postulated goal distraction account of the mechanism. To do so, Study 2 probed for individual differences in weight management goals (e.g., Chernev, 2011; Dhar \& Simonson, 1999; Fishbach \& Zhang, 2008; Wilcox, Vallen, Block, \& Fizsimons, 2009). The main prediction was that the choices of consumers who were strongly concerned with managing their weight and thus possess strong weight management goals would be more affected by the menu presentation variable than the choices of consumers who were less concerned with managing their weight and thus had less strong weight management goals.

This prediction is derived from past work on goals. As mentioned in the preface to Hypothesis 2, past work shows that even when people have goals for a given behavior, whether they will actually behave in line with those goals is affected by environmental factors that are present in the food-consumption context in which they are making their choices. However, those exact same environmental factors have no effect on the choices of people who do not have goals toward the behavior. For instance, people with weight management goals were more likely to choose an apple over a chocolate bar when something in the environment brought their weight management goal to the forefront of their minds-for example, a magazine displaying either a low-calorie option or a particularly high-calorie dessert. However, the food choices of those without weight management goals 
Table 1. The Percentage (\%) of Participants Who Chose Each Possible Combination in Studies 1-3.

\begin{tabular}{llcccc}
\hline Study & Condition & Double Indulgent & Indulgent/Healthy $^{\mathrm{a}}$ & Healthy/Indulgent $^{\mathrm{b}}$ & Double Healthy $^{\text {Dol }}$ \\
\hline Study 1 & Meal & 60.66 & 14.75 & 8.20 & 16.39 \\
(Hamburger, $N=121)$ & A la carte & 38.33 & 38.33 & 11.67 & 11.67 \\
Study 2 & Meal & 31.31 & 23.23 & 22.22 & 23.23 \\
(Waffle, $N=200)$ & A la carte & 23.76 & 13.86 & 32.67 & 29.70 \\
Study 3 & Meal & 40.38 & 23.08 & 23.08 & 13.46 \\
(Waffle, $N=110$ ) & A la carte & 29.31 & 17.24 & 27.59 & 25.86 \\
\hline
\end{tabular}

${ }^{a}$ Indulgent/Healthy signifies the choice of an indulgent main dish and a healthy side item or topping.

${ }^{\mathrm{b}}$ Healthy/Indulgent signifies the choice of a healthy main dish and an indulgent side item or topping.

were not affected by those same health goal cues-they chose the apple and the chocolate bar with the same frequency regardless of whether an environmental cue made the healthy eating goal salient or not (Kruglanski et al., 2002, see also Papies \& Hamstra, 2010).

Because the combination meal versus a la carte choice format is also an environmental manipulation that is predicted to affect the relative salience of healthy eating goals, Hypothesis 2 predicted that a similar effect would occur in the current context. Specifically, the combination meal/a la carte format should impact the food choice behavior of those who are highly concerned with managing their weight, but should have no effect on the choices of those who express little interest in personal weight management. ${ }^{1}$

\section{Participants}

Two hundred adult participants $\left(M_{\text {age }}=34\right.$ years, $43 \%$ female) were recruited from the online panel Mturk. Study 2 used the same procedure as Study 1 with two exceptions. First, participants were asked to choose a waffle and topping combination rather than a burger and side combination. Two waffles-one healthy (a Belgian nine-grain healthy waffle) and one indulgent (a classic Belgian waffle)-were used as the main dishes. In addition, four toppings - two healthy (fresh strawberries and low-fat butter) and two indulgent (chocolate spread and strawberries in whipped cream and syrup)—were used as toppings. All stimuli were presented pictorially. In the meal condition, the eight possible combinations were presented on one page. In the a la carte menu condition, participants selected one of the two waffles first, and then were directed to select one among the four possible toppings.

In addition, following past work on weight management goals and adherence to healthy eating standards

1 The prediction of Hypothesis 2 that people with goals will choose more indulgently following the distracting effects of the combination meal format than after the more simplified and sequential a la carte menu format is derived from past work showing that a primary reason people set weight management goals is to balance out an opposing tendency or desire to tasty and higher calorie food (Stroebe, van Koningsbruggen, Papies, \& Aarts, 2013). If so, then once consumers are distracted from their healthy eating goals by the combination meal condition, they should act in line with their natural desires (e.g., choosing indulgently).
(Chernev, 2011), participants rated how concerned they were with managing their weight on a 7-point scale ( $1=$ Not at all concerned, $7=$ Very concerned). Past work in marketing on dieting and weight management goals (Chernev, 2011) has demonstrated that people who score high on this measure are those who are most likely to be following nutritional guidelines and diets. An additional study conducted for this paper $(N$ $=80$ ) showed a high correlation between this measure of personal concern with weight management and the possession of personal weight management goals (Do you currently have goals to manage your weight?); $r=0.80$.

\section{Results and Discussion}

The key dependent variable was the percentage of people making an indulgent choice for both the waffle and topping choices. This double indulgent choice (i.e., indulgent waffle and indulgent topping) was coded as a " 1 " and all other possibilities were coded as a " 0 ." A logistic regression using the menu presentation format (meal vs. a la carte) as a between-subjects factor and the measured weight-consciousness variable (mean-centered) as a continuous factor revealed significant effects of menu presentation $\left(\chi^{2}=3.81, p=\right.$ $0.05)$ and weight-consciousness $\left(\chi^{2}=9.13, p<0.003\right)$. As predicted, and consistent with Study 1, a greater percent of participants overall selected a double indulgent combination when the menu presented the options in a combination "meal" format (31.31\%) compared to when the menu presented the same options in an a la carte format (23.76\%). However, these effects were qualified by the predicted interaction between the menu presentation variable and participants' dispositional level of weight-consciousness $\left(\chi^{2}=6.01, p\right.$ $<0.02$ ). As predicted by the goal distraction hypothesis, a spotlight analysis based on a fitted logistic regression model showed that the difference between the two menu presentation formats was significant only for participants who held strong weight management goals. High weight conscious consumers were more likely to choose a double indulgent combination when the menu presented the options in the form of combination "meals" (Probability meal $=0.29$ ), than when a similar menu presented the same options as choices in an "a 


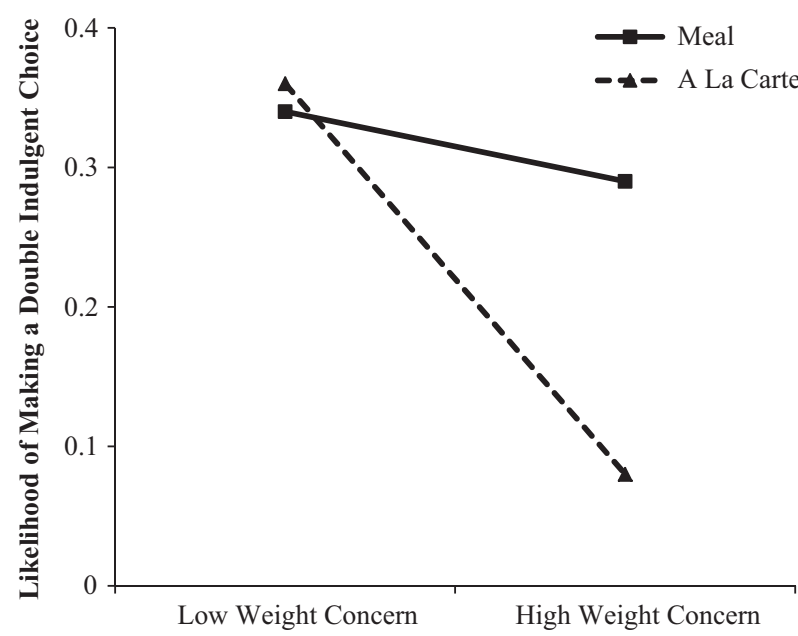

Figure 2. The effects of menu presentation (meal vs. a la carte) and weight concern on double indulgent choicestudy 2. Note: (1) A double indulgent choice is defined as a choice of both an indulgent main dish and an indulgent topping. (2) Low weight concern is $1 \mathrm{SD}$ below the mean, and high weight concern is $1 \mathrm{SD}$ above the mean.

la carte" format (Probability a la carte $=0.08 ; \beta=-1.54$, $Z=-2.78, p<0.006$ ). In contrast, among those who were less personally concerned with managing their weight, the propensity to choose an indulgent/indulgent combination was not affected by the menu presentation format (Probability $_{\text {meal }}=0.34$ vs. Probability a la carte $=0.36 ; \beta=0.12, Z=0.29, p>0.75)$, as shown in Figure 2 .

Table 1 presents the percentage of consumers choosing each of the four possible combinations of main dish and toppings options (indulgent/indulgent; indulgent/healthy; healthy/indulgent; healthy/healthy).

The pattern of these results is consistent with Hypothesis 2 and the goal distraction account. As predicted, the effect of menu format occurred only for consumers who actually held weight management goals; people who were not currently concerned with managing their weight were not affected by the menu presentation.

Study 3 probes for further evidence of the postulated goal distraction mechanism by examining what concepts are cognitively accessible following the presentation of options in a combination presentation format versus a more simplified sequential decision-making one. As outlined earlier, the goal distraction account predicts that the combination format exerts its distracting effects by reducing the salience of healthy eating goals relative to the starker presentation of the a la carte menu format. If so, then concepts related to this goal should be reduced in salience (i.e., be less cognitively accessible) following presentation with combination choices relative to after presentation of the same items in an individualized a la carte fashion.

\section{STUDY 3: IMPLICIT MEASUREMENT OF GOAL DISTRACTION: REDUCED SALIENCE}

Consistent with Hypothesis 1, Study 1 showed that presenting the same food options as combination meals versus a la carte choices increased the likelihood that participants would make unhealthy food choices. Study 2 replicated this effect and began to implicate the goal distraction account by showing that the observed effect is more likely to occur among people who actually hold weight management goals versus not. Study 3 now tests the proposed goal distraction account of the mechanism further by measuring the cognitive accessibility of goal-related concepts following presentation of the two menu formats. Past work shows that the amount of time it takes people to identify words relevant to specific concepts is related to the cognitive accessibility of those concepts (Hogarth, 1987; Payne, Bettman, \& Johnson, 1990). Furthermore, numerous investigations on the topic of goals specifically has shown that response times to goal-relevant concepts and words is a dependable measure of goal activation (e.g., Fishbach et al., 2003; Kruglanski et al., 2002; Stroebe et al., 2008). Thus, if, as was argued earlier, the cross-cutting of healthy and indulgent items into multiple combinations distracts people away from their goals, then words and concepts related to that goal should be less cognitively accessible after seeing items presented in a "combination" format than after seeing the same items were presented in an a la carte presentation style.

To test this, participants in Study 3 completed two different tasks. In the first task, they perused a menu to find a waffle and a topping that they wanted to buy. Half of the participants saw combinations of waffles and toppings and half of the participants saw a menu with a choice of waffles first and a choice of toppings second in a sequential choice format. After looking over their respective menu, participants then completed an attentional task where they identified whether a given string of letters was a word or not as quickly and accurately as possible. Response times to three specific types of stimuli were assessed: (i) goalrelated concepts (i.e., words related to the specific concepts of health and indulgence), (ii) control words (e.g., neutral concepts that were unrelated to the goal at hand), and (iii) nonwords. The key dependent variable was participants' response times to each type of stimuli.

The main prediction was that the "meal" format, with its large number of options with intersecting features, would distract participants from healthy eating goals, thus reducing the activation of conceptually related concepts in their minds. Because both the concept of health itself and its antonym, the to-be-avoided concept of indulgence, are semantically connected in an associative spreading activation framework (e.g., Weiner, Healy, \& Proctor, 2012), the distraction effect should be 
evident on both types of words, resulting in longer response times-indicating more difficulty in processing and less cognitive accessibility of the concepts - to goalrelated words in the combination meal condition than in the a la carte condition. On the other hand, because the menu presentation variable should exert its effects specifically on words that are conceptually related to the goal rather than just any concept overall, participants' response times to the control words should not differ from each other as a function of the menu type they saw. This leads to a prediction of a main effect on processing speed as a function of menu type for the goal-related words and a prediction of no effect on processing speed as a function of menu type for the neutral control words.

\section{Participants, Method, and Design}

Seventy-nine undergraduates at a public university in the Northeast United States completed Study 3 for extra credit in their marketing course $\left(M_{\text {age }}=21\right.$ years, $66 \%$ female). Participants were told that they would participate in several different tasks. The first task was similar to that of Studies 1 and 2; participants considered what combination they would purchase in either a combination meal or an a la carte format. Following procedures used in Laran (2010, study 4B) and Wilcox et al. (2009), participants in Study 3 were simply asked to look over the menu and think about an option that they wanted to purchase rather than make a choice per se, in order to minimize any carryover effects on the reaction time measures that may occur from the act of choosing in and of itself (see Laran, 2010 study 4B and Wilcox et al., 2009 for similar procedures). To examine what was on consumers' minds when they saw the menu and were about to make their choicesthat is, what constructs were accessible at the time of the selection-the immediate next task was an attentional task supposedly designed to investigate participants' ability to identify words quickly and accurately. This task was programed in Media Lab using the software program Direct RT to capture reaction time data. Participants were seated in front of computers in individual cubicle rooms. They first focused on a fixation point $(++)$ on the center of the computer screen and were told that after two seconds, letter strings would be presented one at a time on the screen. Participants were instructed that their task was to identify whether the letter string was a word or not, and they pressed the "A" key for words, and the "L" key for nonwords. Participants performed several practice trials and then responded to the main task. The 10 health-related and 10 indulgence-related words used by Laran (2010); 10 neutral words (cartoon, spoon, picture, flower, sink, forest, notebook, computer, binder and printer); and 30 nonwords were then presented in a random order. Thus, participants responded to 60 trials in total.

\section{Results and Discussion}

Pretest: Selecting Relevant Words. In order to select healthy and indulgence words that were specific to this study, a separate group of 110 undergraduates at the same university completed a study for course credit in their marketing class ( $M_{\text {age }}=21$ years, $37 \%$ female). Participants saw one of the two menus (meal vs. a la carte) as seen by participants in the reaction time main study, and selected which option they would choose. Afterward, all participants rated how much they thought that each of the health and indulgence-related words used by Laran (2010) were relevant to the menu that they looked at ( $1=$ Not much at all, $7=\mathrm{A}$ lot).

Results looking at pretest participants' choice of double indulgent options (i.e., choice of both an indulgent waffle and an indulgent topping) replicated those of Study 2. There was a significant interaction between the manipulated menu presentation variable and the measured weight-consciousness variable $\left(\chi^{2}=4.50, p<\right.$ $0.04)$. Spotlight analyses based on fitted logistic regression models again showed that, as predicted, the menu presentation affected choices only for those who had weight management as a goal (and thus had a goal from which to distract). Specifically, high weight concern individuals were more likely to choose an indulgent waffle plus an indulgent topping when the menu presentation utilized a "meal" format than when it utilized an "a la carte" presentation style (Probability meal $=0.53$ vs. Probability $_{\text {sequential }}=0.22 ; \beta=-1.43, Z=-2.34, p<$ 0.02 ). In contrast, the decisions of those who were low in weight concern were not affected by the menu presentation (Probability $_{\text {meal }}=0.29$ vs. Probability $_{\text {sequential }}$ $=0.38 ; \beta=0.40, Z=0.68, p>0.45$ ). Table 1 presents the percent of pretest consumers choosing each of the four possible main dish and topping combinations.

In order to test the reaction time hypothesis, the five healthy and five indulgence-related words that pretest participants found to be the most relevant to the menus were selected (health-related: fresh $[M=5.39]$, calories $[M=4.55]$, weight [ $M=4.35]$, health $[M=3.93]$, and control $[M=3.87]$; indulgence-related: delicious $[M=$ $6.01]$, enjoy $[M=5.82]$, pleasure $[M=5.64]$, desire $[M=5.55]$, and good $[M=5.51])$.

Main Study Reaction Time Results: Goal Activation. The key dependent measure was participants' response time to the goal-related words. Shorter response time should indicate greater accessibility of a given construct and longer response time should indicate relatively lower accessibility of that construct. Six participants' data were excluded because they pressed only the "A" key or only the "L" key leaving 73 participants for this analysis. Because the meaning of response latencies to incorrect responses is unclear, participants' reaction times to words on which they made errors were eliminated. Before analyses, the data were transformed using a natural log transformation to normalize the distribution of responses (Bargh \& Chartrand, 2000). 
Response times were averaged to generate one score for each category of word (health or indulgence-related or control) for each participant. In order to correct for participant baseline reaction time speed, participants' reaction times to the control words were used as a covariate in the analyses.

The main prediction was that the combination condition would present a distraction to consumers, reducing the accessibility of goal-related concepts (i.e., healthy and indulgent words alike) relative to the a la carte presentation condition. This prediction thus expects that participants' response times to the goal-related words should be slower in the combination condition relative to the a la carte condition. Results from a mixed model ANCOVA using menu format (meal vs. a la carte) as a between-subjects factor, eating goal words (health composite vs. indulgence composite) as a repeated measure, and response time to identifying the control words as a covariate confirmed this prediction. Participants responded more slowly to the eating goal-related words after having seen the combination menu than after having seen a menu utilizing the a la carte format $\left(M_{\text {meal }}=597.15 \mathrm{MS}\right.$ vs. $M_{\text {a la carte }}=$ $572.70 \mathrm{MS} ; F[1,70]=8.51, p<0.005)$. Furthermore, consistent with what would be predicted based on a spreading activation notion, there were no differences as a function of whether the words were related to the health notion itself $\left(M_{\text {meal/healthy }}=601.24\right.$; SD $=95.68$; $\left.M_{\text {a la carte/healthy }}=573.68 ; \mathrm{SD}=75.65\right)$ or to the "to be avoided" concept of indulgence $\left(M_{\text {meal/indulgent }}=593.07\right.$; $\mathrm{SD}=75.64 ; M_{\text {a la carte/indulgent }}=571.72 ; \mathrm{SD}=73.55 ;$ interaction between type of word and menu presentation, $F[1,71]=0.18, p>.65)$. Also as predicted, a separate analysis looking at the control words by themselves showed that the combination presentation condition did not lead participants to be slower to respond to all types of words across the board, their response times to words that were unrelated to eating goals were not differentially affected by the menu presentation (control words: $M_{\text {meal }}=612.46$ milliseconds vs. $M_{\text {a la carte }}$ $=623.91$ milliseconds; $t[71]=0.39, p>0.65)$. Figure 3 presents the mean reaction time to the different types of words as a function of menu presentation condition.

By showing that the combination condition leads to lower accessibility of concepts related to healthy eating goals compared to the sequential a la carte decisionmaking format, these results are consistent with the notion of goal distraction-the intersecting attributes of the combination format appears to produce a situation where goals are neither here nor there-concepts related to them are less salient than they are in an a la carte format, they are less at the forefront of consumers' minds.

\section{GENERAL DISCUSSION}

Across three studies, the current analysis suggests that consumers are more likely to make indulgent choices (e.g., choosing an indulgent /indulgent combination) when choosing among culinary options that are presented in a "meal" or combination format compared to when they make choices among the identical items in an a la carte fashion (Studies 1 and 2). Moreover, consistent with the postulated goal distraction account and past work on environmental cues and adherence to personal goals and standards, this effect occurs more strongly among individuals who actively hold weight management goals and thus have a goal from which to distract (Study 2) and is associated with a lowered cognitive accessibility of goal-related conceptsparticipants show slower response times to health goal related words after exploring a menu offering items as "combination meals" than after seeing the identical items presented in an a la carte format (Study 3).

In a general sense, the present analysis shows how choosing from a large number of options with varying and intersecting key attributes leads to more goal distraction compared with contexts where people make simplified sequential choices along the same key attributes. The present analysis thus complements previous literature on choice and number of options (e.g., Iyengar \& Lepper, 2000; Iyengar et al., 2004) by offering this goal distraction account.

\section{Theoretical Implications}

Though the current analysis tests the proposed goal distraction hypothesis in the context of food choice specifically, an important and pressing consumer issue of interest to policy makers, consumers, and restaurateurs alike, these findings also have more general implications for a wider variety of consumer decisions in the marketplace that are driven by goals. For instance, in addition to weight management, consumers often also have other important goals they try to achieve. Saving money, for instance, or even saving the environment, are commonly held goals among consumers of all backgrounds. The current analysis suggests that people will be better able to achieve these goals and aspirations when choices among options are presented in a "stark," "a la carte-like" sequential choice format than in a combination-like format where the individual attributes or dimensions of a given option are crossed with each other in an intersecting manner in the consideration set. A retailer wanting to encourage consumption of green products, for instance, may want to highlight the "green" versus "regular" distinction on a separate web page choice or even a separate section in the same supermarket aisle rather than simply adding that attribute alongside a larger number of preexisting and intersecting ones in a jumble of products. Regarding product choice more generally, it also suggests a contextual factor that can help consumers reach their goals of choosing ideal products. For example, if a consumer is in the market to purchase a pair of jeans online and has strong preferences regarding the brand, color, and fit, then the consumer will be more likely to purchase the "ideal" pair of jeans if they are presented with a 


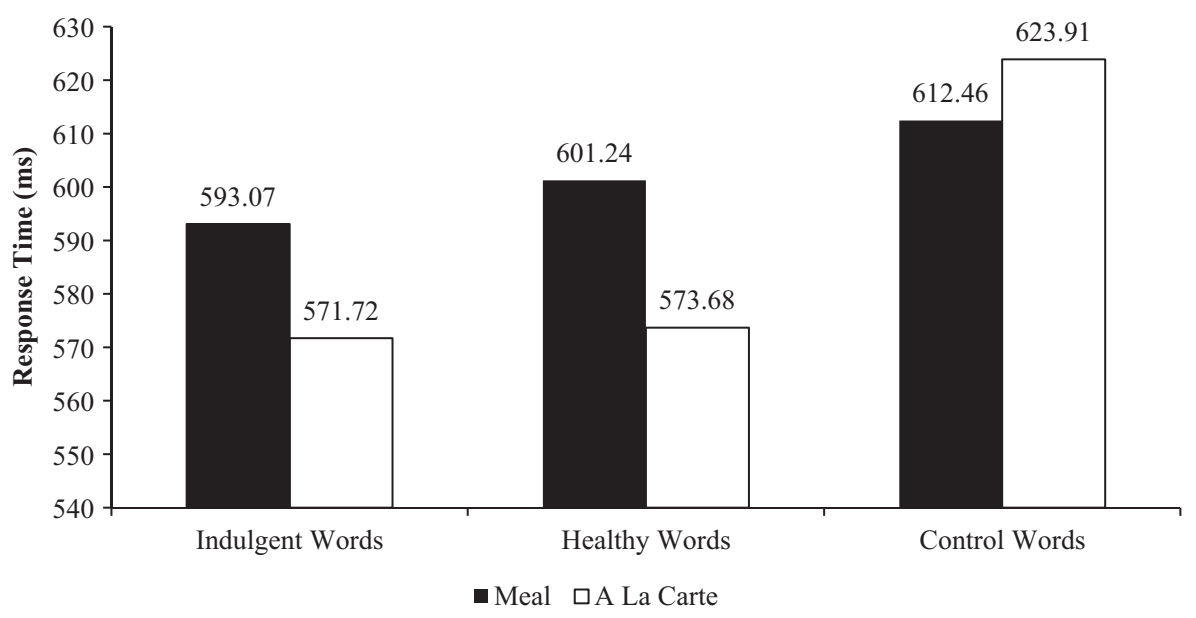

Figure 3. The effect of menu presentation (meal vs. a la carte) on response time to eating-related words (healthy and indulgent) and control words-study 3 .

decision structure that allows choices among the key attributes on search options on the retailer's online website. This would thus mimic the a la carte presentation format and should lead to a more "ideal" purchase. In fact, if the benefits of this simplified choice method were commonly known, perhaps both retailers and consumers alike would waste less time, fewer resources, and avoid frustration processing exchange or return transactions. Of course, this assumes that people have a goal to buy an "ideal" pair of jeans; if that is not a goal, it probably matters less which decision format is used.

In addition to informing the food context specifically, the current results also extend previous work on goals more generally by identifying a context that moderates whether consumers will engage in "highlighting" rather than "balancing" in goal pursuit in situations where there is a trade-off between two goals, in this case health and indulgence (e.g., Dhar \& Simonson, 1999; Fishbach \& Zhang, 2008; Laran, 2010). Specifically, while past work has primarily found "balancing"satisfying one goal now (e.g., indulgence) and another goal later (e.g., health) in contexts where two opposing goals are salient and the person is choosing for him or herself-consumers in the current studies were more likely to highlight-make double indulgent choices-in the meal context than in the a la carte one. The intersecting options appear to distract and reduce the salience of the goal altogether, relative to the stark choice of the sequential format. In demonstrating these results, this work thus adds to a growing amount of research more generally on the contextual factors that affect people's ability to adhere to, and make progress on, their goals.

\section{Practical Implications}

In addition to these more general theoretical considerations, the current work also adds to a growing amount of research on the contextual factors affect people's food choices more specifically by identifying that a ubiquitous yet understudied feature of the food context-the format of the restaurant menu itself-also can either facilitate or distract consumers from healthy eating goals and lead to unrestricted, double indulgent food selection decisions. In doing so, the current research contributes to a burgeoning literature on contextual factors that affect consumers' food-consumption decisions. Specifically, whereas past work in this context has focused on sequential choices (Huber, Goldsmith, \& Mogilner, 2008), or on choices between single healthy and indulgent items (Fishbach \& Zhang, 2008; Okada, 2005; Sela et al., 2009; Wilcox et al., 2009), and has shown that things like "heart-healthy" claims on the menu (Kozup, Creyer, \& Burton, 2003), low-fat labels on food packaging (Wansink \& Chandon, 2006), and health claims in fast-food branding (i.e., Subway's sandwich vs. McDonald's sandwich; Chandon \& Wansink, 2007) can increase the likelihood of consuming indulgent foods, the current analysis builds on this work by demonstrating the fact that something as innocuous (and ubiquitous) as ordering procedures can also affect the likelihood of choosing indulgent options. Consumers make better health decisions when they choose food items one by one rather than when they choose them as combinations (e.g., meals), especially when they endorse healthy eating as a personal goal.

\section{CONCLUSION}

In sum, the current research shows how consumers can become more distracted from their goals when choosing among a large number of options with varying intersecting key attributes, compared to situations where they are making simplified sequential decisions among the same choice alternatives along key attributes. Consequently, consumers make choices that are less 
consistent with their goals in the former numerous and intersecting options choice format than in the latter simplified sequential choice format. Moreover, this research explores these dynamics in the ubiquitous and important context of food selection. Presenting items as "meals" or combinations leads people to make more indulgent decisions relative to a menu context where the same items are presented in an a la carte fashion. Thus, while culinary options such as "value meals" have been criticized for leading to extra caloric intake through phenomena such as "supersizing," the current research actually suggests that the mere act of presenting food items in a combination format to begin with can in and of itself contribute to unhealthy food choices.

\section{REFERENCES}

Bargh, J., \& Chartrand, T. L. (2000). The mind in the middle: A practical guide to priming and automaticity research. In $\mathrm{H}$. T. Reis \& C. M. Judd (Eds.), Handbook of research methods in social and personality psychology (1st ed., pp. 253-285). New York: Cambridge University Press.

Broniarczyk, S. M., \& Griffin, J. (2014). Decision difficulty in the age of consumer empowerment. Journal of Consumer Psychology, 24, 608-625.

Chandon, P., \& Wansink, B. (2007). The biasing health halos of fast-food restaurant health claims: Lower calorie estimates and higher side-dish consumption intentions. Journal of Consumer Research, 34, 301-314.

Chernev, A. (2011). The dieter's paradox. Journal of Consumer Psychology, 21, 178-183.

Dhar, R., \& Simonson, I. (1999). Making complementary choices in consumption episodes: Highlighting versus balancing. Journal of Marketing Research, 36, 29-44.

Fishbach, A., \& Zhang, Y. (2008). Together or apart: When goals and temptations complement versus compete. Journal of Personality and Social Psychology, 94, 547-559.

Fishbach, A., Friedman, R. S., \& Kruglanski, A. W. (2003). Leading us not into temptation: Momentary allurements elicit overriding goal activation. Journal of Personality and Social Psychology, 84, 296-309.

Foxman, E. R., Berger, P. W., \& Cote, J. A. (1992). Consumer brand confusion: A conceptual framework. Psychology \& Marketing, 9, 123-141.

Herman, C. P., \& Polivy, J. (1983). A boundary model for the regulation of eating. Psychiatric Annals, 13, 918-927

Hogarth, R. M. (1987). Judgement and choice: The psychology of decision. Oxford, England: John Wiley \& Sons.

Huber, J., Goldsmith, K., \& Mogilner, C. (2008). Reinforcement versus balance response in sequential choice. Marketing Letters, 19, 229-229.

Huffman, C., \& Kahn, B. E. (1998). Variety for sale: Mass customization or mass confusion? Journal of Retailing, 74, 491-513.

Iyengar, S. S., \& Lepper, M. R. (2000). When choice is demotivating: Can one desire too much of a good thing? Journal of Personality and Social Psychology, 79, 995-1006.

Iyengar, S. S., Huberman, G., \& Jiang, W. (2004). How much choice is too much? Contributions to $401(\mathrm{k})$ Retirement Plans. In O. S. Mitchell \& S. P. Utkus (Eds.), Pension design and structure: New lessons from behavioral finance (1st ed., pp. 83-95). Oxford, UK: Oxford University Press.

Jacoby, J., Speller, D. E., \& Berning, C. K. (1974). Brand choice behavior as a function of information load: Replication and extension. Journal of Consumer Research, 1, 33-42.

Jacoby, J., Speller, D. E., \& Kohn, C. A. (1974). Brand choice behavior as a function of information load. Journal of Marketing Research, 1, 63-69.

Jia, J. S., Shiv, B., \& Rao, S. (2014). The product-agnosia effect: How more visual impressions affect product distinctiveness in comparative choice. Journal of Consumer Research, 41, 342-360.

Kozup, J. C., Creyer, E. H., \& Burton, S. (2003). Making healthful food choices: The influence of health claims and nutrition information on consumers' evaluations of packaged food products and restaurant menu items. Journal of Marketing, 67, 19-34.

Kruglanski, A. W., Shah, J. Y., Fishbach, A., Friedman, R., Chun, W. Y., \& Sleeth-Keppler, D. (2002). A theory of goal systems. Advances in Experimental Social Psychology, 34, 331-378.

Laran, J. (2010). Goal management in sequential choices: Consumer choices for others are more indulgent than personal choices. Journal of Consumer Research, 37, 304-314.

Mitchell, V., Walsh, G., \& Yamin, M. (2005)., Toward a conceptual model of consumer confusion. Advances in Consumer Research, 32, 143-150.

Okada, E. M. (2005). Justifying the hedonic and the effects on fun versus practical consumption. Journal of Marketing Research, 42, 43-53.

Papies, E. K., \& Hamstra, P. (2010). Goal priming and eating behavior: Enhancing self-regulation by environmental cues. Health Psychology, 29, 384-388.

Payne, J. W., Bettman, J. R., \& Johnson, E. J. (1990). The adaptive decision maker: Effort and accuracy in choice. In R. M. Hogarth (Ed.), Insights in decision making: A tribute to Hillel J. Einhorn (pp. 129-153). Chicago: University of Chicago Press.

Sela, A., Berger, J., \& Liu, W. (2009). Variety, vice and virtue: How assortment size influences option choice. Journal of Consumer Research, 35, 941-950.

Stroebe, W., Mensink, W., Aarts, H., Schut, H., \& Kruglanski, A. W. (2008). Why dieters fail: Testing the goal conflict model of eating. Journal of Experimental Social Psychology, $44,26-36$.

Stroebe, W., van Koningsbruggen, G. M., Papies, E. K., \& Aarts, H. (2013). Why most dieters fail but some succeed: A goal conflict model of eating behavior. Psychological Review, 120, 110-138.

Wansink, B., \& Chandon, P. (2006). Can "low-fat" nutrition labels lead to obesity? Journal of Marketing Research, 43, 605-617.

Weiner, I.B., Healy, A.F., \& Proctor, R.W. (Eds.). (2012). Handbook of psychology-Volume 4: Experimental psychology. Hoboken, NJ: Wiley.

Wilcox, K., Vallen, B., Block, L., \& Fizsimons, G. J. (2009). Vicarious goal fulfilment: When the mere presence of a healthy option leads to an ironically indulgent decision. Journal of Consumer Research, 36, 380-393.

Correspondence regarding this article should be sent to: Yong Kyu Lee, Assistant Professor, Department of Business and Economics, School of Business and Information Systems, York College, The City University of New York (CUNY), 94-20 Guy R. Brewer Blvd, Jamaica, NY 11451 (ylee1@york.cuny.edu). 